\title{
Errata
}

\section{Calculation of Optinum Geometries and Force Fields by the CNDO/Force Method}

Meenakshisundaram Kanakavel, Jayaraman Chandrasekhar, Sankaran Subramanian and Surjit Singh

Theoret. Chim. Acta (Berl.) 43, 185-196 (1976).

Ref. [30] should read: Schachtschneider, J. H., in: Vibrational spectra of polyatomic molecules VI, Tech. Reports No. 57-65. Shell Development Co., California, USA 1964

Received July 24, 1978 